R. A. Blumenthal

Nagoya Math. J.

Vol. 83 (1981), 197-201

\title{
RIEMANNIAN HOMOGENEOUS FOLIATIONS WITHOUT HOLONOMY
}

\author{
ROBERT A. BLUMENTHAL
}

\section{§1. Introduction}

Let $M$ be a compact connected $C^{\infty}$ manifold with a smooth Riemannian foliation $\mathscr{F}$. That is, $(M, \mathscr{F})$ admits a bundle-like metric in the sense of [7]. In [4] it is shown that if all leaves of $\mathscr{F}$ are closed without holonomy, then the space of leaves $M / \mathscr{F}$ of the foliation is a manifold and the natural projection $M \rightarrow M / \mathscr{F}$ is a locally trivial fibre space. In the present work we show that for certain of the Riemannian homogeneous foliations, holonomy is the only obstruction to the foliation being a fibration.

Let $G / K$ be a simply connected, even dimensional, positively curved Riemannian homogeneous space of a compact connected Lie group $G$ and let $\mathscr{F}$ be a homogeneous $G / K$-foliation of a compact connected manifold $M$. For example, $\mathscr{F}$ is a codimension $2 q$ elliptic (i.e., homogeneous $\left.S O(2 q+1) / S O(2 q) \cong S^{2 q}-\right)$ foliation. Then $\mathscr{F}$ is cohomologically a fibration in the sense that the base-like cohomology algebra of the foliated manifold $(M, \mathscr{F})$ is isomorphic to the de Rham cohomology algebra of $G / K$ [3]. The main result of this paper asserts that if $\mathscr{F}$ has no holonomy, then it is actually a fibration.

(1.1) TheoREM. If $\mathscr{F}$ is without holonomy, then $M$ fibres over $G / K$ with the leaves of $\mathscr{F}$ as fibres.

\section{§2. Riemannian Homogeneous Foliations}

In this section we prove (1.1) and use its proof to elucidate further properties of Riemannian homogeneous foliations.

Let $G / K$ be a connected homogeneous space on which $G$ acts effectively and let $\mathscr{F}$ be a homogeneous $G / K$-foliation of a connected manifold $M$. That is, $\mathscr{F}$ is defined by a $G / K$-cocycle $\left\{\left(U_{\alpha}, f_{\alpha}, \lambda_{g_{\alpha \beta}}\right)\right\}_{\alpha, \beta \in A}$ where

Received November 12, 1979. 
i) $\left\{U_{\alpha}\right\}_{\alpha \in A}$ is an open cover of $M$

ii) $f_{\alpha}: U_{\alpha} \rightarrow G / K$ is a submersion defining $\mathscr{F} \mid U_{\alpha}$

iii) $f_{\alpha}=\lambda_{g_{\alpha \beta}} \circ f_{\beta}$ on $U_{\alpha} \cap U_{\beta}$ where $g_{\alpha \beta} \in G$ and $\lambda_{g_{\alpha \beta}}$ denotes the diffeomorphism of $G / K$ which sends $a K$ into $g_{\alpha \beta} a K$.

To prove the theorem, we recall a construction from [2] which gives a useful realization of the holonomy group of a leaf of $\mathscr{F}$.

Let $P=\left\{\left[\lambda_{g} \circ f_{\alpha}\right]_{x}: x \in U_{\alpha}, \alpha \in A, g \in G\right\}$, where $\left[\lambda_{g} \circ f_{\alpha}\right]_{x}$ denotes the germ of $\lambda_{g} \circ f_{\alpha}$ at $x$ and let $\pi: P \rightarrow M$ be defined by $\pi\left(\left[\lambda_{g} \circ f_{\alpha}\right]_{x}\right)=x$. Then $P$ possesses a well-defined topology and differentiable structure such that $\pi: P \rightarrow M$ is a smooth regular covering. Moreover, the evaluation map $F: P \rightarrow G / K$ defined by $F\left(\left[\lambda_{g} \circ f_{\alpha}\right]_{x}\right)=\lambda_{g}\left(f_{\alpha}(x)\right)$ is a smooth submersion constant along the leaves of $\pi^{-1}(\mathscr{F})$ where $\pi^{-1}(\mathscr{F})$ denotes the foliation of $P$ whose leaves are the connected components of the inverse images under $\pi$ of the leaves of $\mathscr{F}$. By choosing a connected component of $P$, we may assume that $P$ is connected. This connected regular covering gives rise to a homomorphism $\Phi: \pi_{1}\left(M, x_{0}\right) \rightarrow G$ such that $\Gamma=$ image $(\Phi)$ is the group of covering transformations and such that the diagram

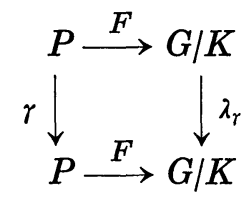

is commutative for each $\gamma \in \Gamma$.

Let $L$ be a leaf of $\mathscr{F}$ and choose a leaf $L^{\prime}$ of $\pi^{-1}(\mathscr{F})$ which projects to $L$. Then the holonomy group of $L$ is isomorphic to $\Gamma_{L^{\prime}}=\left\{\gamma \in \Gamma: \gamma\left(L^{\prime}\right)\right.$ $\left.=L^{\prime}\right\}$ and thus $\pi \mid L^{\prime}: L^{\prime} \rightarrow L$ is a regular covering with the holonomy group of $L$ as its group of covering transformations.

If $K$ is compact, then $M$ admits a bundle-like metric (whence $\mathscr{F}$ is called a Riemannian homogeneous foliation) such that the lifted metric on $P$ is bundle-like with respect to the foliation defined by the submersion $F$. Thus if $M$ is also compact, we have that $F: P \rightarrow G / K$ is a locally trivial fibre space [5]. (We remark that since an isometry of a connected Riemannian manifold is determined by its value and differential at a point, this construction remains valid for a foliation defined by an $N$-cocycle $\left\{\left(U_{\alpha}, f_{\alpha}, g_{\alpha \beta}\right)\right\}_{\alpha, \beta \in A}$ where $N$ is a connected Riemannian manifold and each $g_{\alpha \beta}$ extends to an isometry of $N$.)

Assume now that $M, \mathscr{F}$, and $G / K$ satisfy the hypotheses of (1.1). Fix 
an orientation on $G / K$ invariant under the action of $G$. Let $\gamma \in \Gamma$. Then $\lambda_{r}$ is an orientation-preserving isometry of $G / K$ and hence has a fixed point $y$ [6]. It is here that we have used the assumption in Theorem (1.1) that $G / K$ is an even dimensional, positively curved Riemannian homogeneous space of the compact connected Lie group $G$. Since $F: P \rightarrow G / K$ is a fibration over a simply connected manifold, we have that the space of leaves of $\pi^{-1}(\mathscr{F})$ is diffeomorphic to $G / K$ and hence there exists a unique leaf $L_{0}^{\prime}$ of $\pi^{-1}(\mathscr{F})$ such that $F\left(L_{0}^{\prime}\right)=y$. Now $F\left(\gamma\left(L_{0}^{\prime}\right)\right)=\lambda_{r}\left(F\left(L_{0}^{\prime}\right)\right)=\lambda_{r}(y)=$ $y=F\left(L_{0}^{\prime}\right)$ and hence $\gamma\left(L_{0}^{\prime}\right)=L_{0}^{\prime}$. Since $\Gamma_{L_{0^{\prime}}}$ is isomorphic to the holonomy group of the leaf $L_{0}=\pi\left(L_{0}^{\prime}\right)$ of $\mathscr{F}$, it follows that $\gamma$ is the identity transformation. Hence $\Gamma$ is trivial and so $M$ is diffeomorphic to $P$ whence $F: M \rightarrow G / K$ is a fibration with the leaves of $\mathscr{F}$ as fibres.

(2.1) Corollary. Let $G / K$ be a simply connected, even dimensional, positively curved Riemannian homogeneous space of a compact connected Lie group $G$ and let $\mathscr{F}$ be a one-dimensional homogeneous $G / K$-foliation of a compact connected manifold $M$. Then $\mathscr{F}$ has a compact leaf and $\pi_{1}(M)$ has polynomial growth of degree $\leq 1$.

Proof. If $\mathscr{F}$ is without holonomy, then by (1.1) the leaves of $\mathscr{F}$ are the fibres of a fibration $M \rightarrow G / K$ and hence all the leaves are circles. Since $G / K$ is simply connected, the exact homotopy sequence of this fibration gives a surjection $\pi_{1}\left(S^{1}\right) \rightarrow \pi_{1}(M)$ whence $\pi_{1}(M)$ has polynomial growth of degree $\leq 1$. If $\mathscr{F}$ has a leaf $L$ whose holonomy group is nontrivial, then $L$ is diffeomorphic to $S^{1}$. Moreover, since $L$ is compact, the image of its fundamental group in $\pi_{1}(M)$ is a subgroup of finite index [2] and hence $\pi_{1}(M)$ has polynomial growth of degree $\leq 1$ [1].

(2.2) Proposition. Let $\mathscr{F}$ be a codimension 2 transversely Euclidean (homogeneous $S O(2) \cdot R^{2} / S O(2) \cong R^{2}$ ) foliation of a compact connected manifold $M$. If $\mathscr{F}$ is without holonomy, then $M$ fibres over $T^{2}$.

Proof. In this case $\Gamma$ is a subgroup of $S O(2) \cdot R^{2}$, the group of rigid motions of the plane. Since $\mathscr{F}$ is without holonomy, it follows that $\Gamma$ acts freely on $\boldsymbol{R}^{2}$ and hence is a group of translations. If $(x, y)$ denotes the standard coordinates on $R^{2}$, then $d x$ and $d y$ are linearly independent translation-invariant one-forms and hence there exist smooth linearly independent one-forms $\omega_{1}$ and $\omega_{2}$ on $M$ such that $\pi^{*} \omega_{1}=F^{*}(d x), \pi^{*} \omega_{2}=F^{*}(d y)$. Moreover, $\omega_{1}$ and $\omega_{2}$ are closed and so $M$ fibres over $T^{2}$ [8]. 
We now apply the above construction to study the existence of compact leaves for a class of Riemannian homogeneous foliations. Let $G / K$ be a compact simply connected Riemannian symmetric space with nonzero Euler characteristic where $G=I_{0}(G / K)$.

(2.3) Proposition. Let $M$ be a compact manifold with solvable fundamental group. Then every homogeneous $G / K$-foliation of $M$ has a compact leaf.

Proof. The image $\Gamma$ of the homomorphism $\Phi: \pi_{1}\left(M, x_{0}\right) \rightarrow G$ is a solvable subgroup of the compact Lie group $G$ and so its closure is a compact solvable Lie subgroup of $G$. Let $H$ be the connected component of the identity of $\bar{\Gamma}$. Then $H$ is a toral subgroup. Since the Euler characteristic of $G / K$ is nonzero, $G$ and $K$ have the same rank [9] and hence $H$ is contained in some conjugate of $K$. Thus there exists a point $y$ of $G / K$ fixed under the action of $H$ and hence, since $H$ is a subgroup of $\bar{\Gamma}$ of finite index, the orbit of $y$ under the action of $\Gamma$ is a finite set of points $y=y_{1}, y_{2}, \cdots, y_{r}$. For each $i=1, \cdots, r$ let $L_{i}$ be the unique leaf of $\pi^{-1}(\mathscr{F})$ such that $F\left(L_{i}\right)=y_{i}$. Then $L_{1}, \cdots, L_{r}$ all project via $\pi$ to the same leaf $L$ of $\mathscr{F}$ and, since $\pi^{-1}(L)=\cup_{i=1}^{r} L_{i}$ is a closed subset of $P$, it follows that $L$ is compact.

Recall that by a codimension $q$ elliptic foliation of a manifold $M$ we mean a homogeneous $G / K$-foliation of $M$ where $G=S O(q+1), K=S O(q)$, $G / K \cong S^{q}$.

(2.4) CoROllaRY. Every codimension $2 q$ elliptic foliation of a compact manifold with solvable fundamental group has a compact leaf.

\section{REFERENCES}

[1] H. Bass, The degree of polynomial growth of finitely generated nilpotent groups, Proc. London Math. Soc., (3) 25 (1972), 603-614.

[ 2 ] R. Blumenthal, Transversely homogeneous foliations, Annales de l'Institut Fourier 29 (1979), 143-158.

[ 3 ] - The base-like cohomology of a class of transversely homogeneous foliations, Bulletin des Sciences Mathématiques, 104 (1980), 301-303.

[4] R. Hermann, On the differential geometry of foliations, Annals of Math., (3) 72 $(1960), 445-457$.

[5] - A sufficient condition that a mapping of Riemannian manifolds be a fibre bundle, Proc. Amer. Math. Soc., 11 (1960), 236-242.

[6] S. Kobayashi, Transformation groups in differential geometry, Ergebnisse der Mathematik und ihrer Grenzgebiete (70) Springer-Verlag, Berlin, 1972. 
[7] B. Reinhart, Foliated manifolds with bundle-like metrics, Annals of Math., (1) 69 (1959), 119-132.

[8] D. Tischler, On fibering certain foliated manifolds over $S^{1}$, Topology $9(1970)$, 153-154.

[ 9 ] J. Wolf, Spaces of constant curvature, McGraw-Hill, New York, 1967.

Université des Sciences et Techniques de Lille I

U.E.R. de Mathématiques Pures et Appliquées

B.P. 36-59650 Villeneuve d'Ascq

France

Department of Mathematics

St. Louis University

St. Louis, Missouri 63103

U.S.A. 